\title{
Implementación del uso de la Radio Definida por Software como nuevo recurso didáctico para el diseño aplicado en Ingeniería Telemática a partir del Aprendizaje Basado en Problemas
}

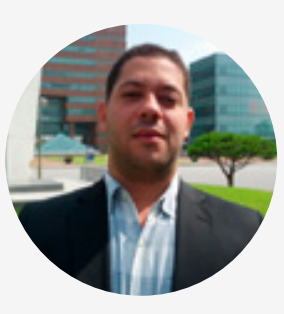

DR. VÍCTOR MANUEL GONZÁLEZ HOLGUÍN*

\author{
The usage of Software Define Radio as \\ an Educational Resource for Teaching \\ Tel-ecommunication Design Principles \\ with Problem Based Learning
}

Recibido: 13 de mayo de 2019 | Aprobado: 14 de junio de 2019

\section{Resumen}

La educación en ingeniería debe ir a la par con las herramientas que se utilizan en la industria. Se deben elegir recursos y metodologías que promuevan aprendizajes activos que ayuden a desarrollar las competencias específicas requeridas en el mercado laboral. Este artículo documenta el desarrollo de un plan de implementación para el cambio al uso de software para el diseño de sistemas de comunicación, en el cual se pudo re-enfocar las actividades de laboratorio, concentrándose en el desarrollo de las competencias específicas relacionadas al diseño de sistemas de comunicación a partir de las estrategias del Aprendizaje Basado en Problemas. El estudio responde a la metodología de investigación-acción. Se puede observar que la metodología de enseñanza-aprendizaje utilizada en conjunto con el recurso didáctico tuvo un impacto directo en el mejoramiento de la capacidad de solución de problemas de los estudiantes y el mejoramiento en el desempeño de sus diseños. Estos cambios han aumentado la motivación en las tareas encomendadas y, además, permiten una transferencia de las habilidades adquiridas al ámbito laboral de acuerdo a los objetivos del aprendizaje de las competencias propias de la carrera.

Palabras clave: radio definida por software; sistema de comunicación; diseño; ABP; recurso

\section{Abstract}

There is a need to align the tools used in engineering education to those found in the industry. To use these tools universities need to implemente active teaching methodologies that help with the development of their students outcome requirements. In this article we document the usage of the action research methodology for the development of a strategy to implement software based design in communication systems. This strategy refocused the laboratories activities in developing design skills in students using the fundamentals of problem based learning. The usage of problem based learning with software define radio allowed the development of problem solving skills in our students. At the same time, the students could design more complex solutions with higher performance in comparison with previous classes. Finally, this new strategy increase our students motivation in the area of study.

Keywords: software-defined radio; communication system; design; PBL; resource

\footnotetext{
* Doctor en Tecnología de la Información y Telecomunicación por Korea Advanced Institute of Science and Technology (KAIST). Es profesor investigador a tiempo completo en la Pontificia Universidad Católica Madre y Maestra. Además, se ha desempeñado como revisor invitado en distintas revistas y conferencias académicas internacionales. Para contactar al autor: victorgonzalez@pucmm.edu.do
}

ISSN (en línea): 1814-4152 / Sitio web: http://cuaderno.pucmm.edu.do

CÓMO CITAR: González, V. (2019). Implementación del uso de la Radio Definida por Software como nuevo recurso didáctico para el diseño aplicado en Ingeniería Telemática a partir del Aprendizaje Basado en Problemas. Cuaderno de Pedagogía Universitaria, Vol. 16, n. ${ }^{\circ} 32$, julio-diciembre, pp. 60-69 


\section{Introducción}

En la educación superior actual es importante utilizar recursos didácticos que permitan desarrollar las competencias específicas de forma más efectiva, creando un vínculo directo con las prácticas profesionales. Para crear este vínculo se necesitan metodologías de enseñanza aprendizaje activas que incentiven el desarrollo de competencias complejas como la toma de decisiones y solución de problemas (Sanz de Acedo, 2010). El Aprendizaje Basado en Problemas (ABP) es una metodología altamente utilizada para el desarrollo de competencias relacionadas al diseño, ya que un problema conlleva una tarea compleja cuya solución se asocia a la necesidad de diseñar (Burgess, 2004). El diseño y la resolución de problemas son competencias entrelazadas debido a que ambas requieren de pasos similares que van desde la identificación a la evaluación.

En la Ingeniería Telemática tradicionalmente se han desarrollado las competencias necesarias con el diseño de sistemas de comunicaciones utilizando componentes electrónicos. El uso de estos componentes agrega pasos adicionales que aumentan el tiempo de elaboración de las prácticas que deben lograr los estudiantes al conectar cables entre componentes y solucionar problemas que ocurren en las señales entre dichos componentes. Aunque estas habilidades son importantes, no corresponden a las propias del diseño de sistemas de comunicación ni al paradigma actual en el ámbito profesional.

La introducción del uso de la Radio Definida por Software (SDR, por sus siglas en inglés) en las asignaturas relacionadas a sistemas de comunicación permite que se desarrollen las competencias específicas de forma más efectiva, debido a que aproxima al estudiante a los métodos de diseño utilizados en el ámbito profesional de la telecomunicación (García, Barros, Gusso, Pedroso y Barbin, 2012; Núñez, Mascareñas y Pérez-Iñigo, 2016). El SDR es el paradigma actual en el que la industria desarrolla y prueba los nuevos estándares que definen los sistemas de comunicación inalámbrica que utilizamos (Akeela y Dezfouli, 2018).

El uso de SDR ya es común en universidades extranjeras (Aguayo, Dietrich, Sayed, Volos, Gaeddert, Robert y Kragh, 2009; Küçük, 2018; Núñez et al, 2016). Nuestro estudio es el primer caso que se documenta en la República Dominicana. Aunque existen múltiples artículos del uso de SDR en las universidades, pocos han descrito la metodología de enseñanza-aprendizaje para su implementación. En este artículo presentamos un proceso de investigación-acción llevado a cabo con el objetivo de re-orientar la asignatura de Electrónica de Comunicación al desarrollo de la competencia en diseño de sistemas de comunicación. Se utilizó la metodología del ABP para el desarrollo de la competencia de diseño y el SDR como recurso didáctico.

El objetivo de este estudio es documentar el desarrollo de un plan de implementación para realizar un cambio al uso de software para el diseño de sistemas de comunicación, en el cual se pudo re-enfocar las actividades de laboratorio, concentrándose en el desarrollo de las competencias específicas relacionadas al diseño de sistemas de comunicación mediante el uso de resolución de problemas a partir de la metodología del ABP. En las siguientes secciones se exponen los fundamentos del Aprendizaje Basado en Problemas y su aplicación en ingeniería; el concepto de SDR y sus componentes; luego se describe el proceso de investigación-acción y las etapas y evaluación de los problemas planteados. Finalmente, se presentan las implicaciones y conclusiones más relevantes.

Las preguntas que provocaron este cambio de recurso y metodología de enseñanza-aprendizaje fueron:

- ¿Por qué los componentes electrónicos tradicionales no son los más adecuados para desarrollar las competencias específicas de diseño de los ingenieros telemáticos?

- ¿Qué efecto tiene en los estudiantes el planteamiento de problemas estructurados y a partir de metodologías tradicionales, en comparación con problemas abiertos, mal definidos y de forma desestructurada?

- ¿Cómo cambia el rol del docente al implementar una metodología activa para el diseño a partir de problemas reales?

\section{Aprendizaje Basado en Problemas}

El Aprendizaje Basado en Problemas como es conocido, es una evolución de una innovación en el currículo de medicina de la Universidad de McMaster en Canadá en la década del 60 del siglo XX. Los cambios se iniciaron por las necesidades cognitivas de los profesionales de la medicina que los métodos tradicionales no estaban supliendo. En las décadas de los 80 y 90 la metodología ABP fue adoptada en múltiples escuelas de medicinas y se estableció 
como un método de enseñanza-aprendizaje en Norteamérica y Europa (Savery, 2006). En la actualidad, esta metodología se ha implementado en casi todas las áreas del saber, entre ellas en la ingeniería.

La efectividad del ABP se ha podido probar en varias disciplinas de ingeniería. El principio fundamental de esta metodología es exponer al estudiante a un problema real que el estudiante pueda resolver y finalmente presentar los conceptos generalizados que fundamentan dicho problema. Esta metodología funciona en tres etapas iterativas en las cuales el estudiante está activamente involucrado en su aprendizaje y el profesor debe fungir como guía del estudiante. En la primera etapa el estudiante debe identificar los conocimientos necesarios para resolver el problema expuesto. Luego, el estudiante se basa en estudios auto-dirigidos para cubrir las brechas de conocimiento detectadas en la etapa anterior. Por último, aplica el conocimiento adquirido para resolver el problema original. Con cada problema expuesto inicia este ciclo iterativo (Perrenet, Bouhuijs y Smits, 2000). "Escoger y plantear un problema relevante y complejo es acción definitiva en la estrategia ABP, ya que la solución de la mayoría de los problemas toma un tiempo generalmente largo" (Gómez, 2005, p.12).

El ABP responde a los objetivos educacionales de: (1) adquirir conocimiento que se va a utilizar en un contexto profesional, (2) desarrollar competencias que mejoran el conocimiento individual y el desarrollo de la capacidad de solucionar problemas (Perrenet et al., 2000). Grolinger (2011) destacó cómo la implementación de ABP en la educación en ingeniería ha ayudado a lograr las demandas profesionales de la industria cuando los problemas expuestos en el aula son similares a los que encontrarán en el ámbito profesional. Esto también ayuda a construir en el estudiante una identidad profesional del área de estudio (Tan, Van der Molen, y Schmidt, 2016).

En un estudio comparativo entre ABP y clases magistrales dentro de un curso de ingeniería eléctrica se pudo identificar que los estudiantes expuestos al ABP obtuvieron un aprendizaje doblemente mayor que los expuestos al método tradicional (Yadav, Subedi, Lundeberg y Bunting, 2011). Patil (2016) obtuvo resultados similares en un curso de diseño de sistemas embebidos, los estudiantes de la sección que utilizó la metodología del ABP obtuvieron un promedio mayor del puntaje de evaluación total que los estudiantes en la sección tradicional.
Otros estudios sobre el uso del ABP, dentro de la enseñanza de la ingeniería, han identificado que los estudiantes desarrollan motivaciones intrínsecas que permiten un mayor aprendizaje (Harun, Yusof, Jamaludin y Hassan, 2012). Esta metodología tiene un efecto directo en la habilidad de pensamiento y la adquisición de conocimiento (Masek y Yamin, 2012) e incentiva el empoderamiento del estudiante, lo cual permite completar actividades complejas que mejoran su rendimiento (El-adaway, Pierrakos y Truax, 2014).

La implementación de esta metodología requiere una intervención especial por parte del profesor, el cual debe ser un facilitador en las discusiones del problema para llevar al estudiante a la búsqueda de información relevante para una solución apropiada (Hmelo-Silver, 2013). Dentro de este contexto, un alto conocimiento del contenido por parte del profesor no tiene una correlación directa al aprendizaje del estudiante (Leary, Walker y Shelton, 2013), sin embargo, permite la selección, construcción y aplicación de "buenos" problemas para el aprendizaje del estudiante (Bejarano y Lirio, 2008). El instructor, además del conocimiento técnico, debe tener la capacidad de realizar preguntas apropiadas en momentos idóneos para llevar una discusión sostenible del problema y crear un escenario propicio para el aprendizaje. Es de gran importancia que el profesor tenga conocimiento de la metodología del ABP y un plan de implementación (Rico y Ertmer, 2015).

El plan de implementación debe considerar el proceso de identificación y análisis del problema por parte del estudiante, al igual que el proceso de solución del mismo (Ertmer y Stepich, 2005). Para proponer problemas considerados "buenos", el profesor debe asegurar que estos estén conectados al desarrollo de las competencias a desarrollar por los estudiantes y a la realidad del ámbito profesional. Estos problemas deben ser abiertos y con múltiples soluciones, presentados de forma desestructurada y mal definidos (Bejarano y Lirio, 2008).

\section{La Radio Definida por software}

El propósito principal dentro del paradigma de Radio Definido por Software (SDR) es realizar las distintas etapas del procesamiento de la señal a nivel de software sobre componentes electrónicos reconfigurables (Jondral, 2005). El problema del procesamiento utilizando los métodos anteriores basados en hardware es la poca adaptabilidad que presentan los circuitos electrónicos. Estos circuitos 
nos limitan el uso del esquema de modulación, las frecuencias de operación y el ancho de banda, entre otros aspectos del sistema. Dentro de un ambiente de prueba y desarrollo, el uso de equipos convencionales limita el desarrollo y prueba de múltiples estándares o diferentes versiones de un estándar, debido al alto costo de tener equipos individuales para cada estándar y/o versión (Akeela y Dezfouli, 2018). Por otro lado, si el estudiante diseña e implementa cada estándar y/o versión utilizando componentes electrónicos para el procesamiento, pierde mucho tiempo en el ensamblaje de los distintos circuitos (Garcia et al., 2012).

La arquitectura de SDR se divide en la antena, la interfaz de radiofrecuencia, la interfaz de procesamiento digital y la interfaz del procesamiento de la señal (Patton, 2007). El orden de las interfaces dependerá si es un transmisor o un receptor, como se puede observar en la figura 1.

En el mercado existen varias herramientas de software y de hardware programables que se pueden utilizar para implementar una plataforma SDR. Akeela y Dezfouli (2018) realizan una extensiva revisión de la literatura en el tema y presentan las distintas arquitecturas existentes y las herramientas de software y hardware que se pueden utilizar. Dentro de las comparativas realizadas en las herramientas de software, GNU Radio presenta una ventaja por su licenciamiento de código libre.
GNU Radio es una herramienta de desarrollo de software especializada para SDR. Dentro del paquete se incluyen múltiples bloques de procesamiento de señal. Es una plataforma utilizada tanto en la industria como en la academia (García et al., 2012). Otra de las ventajas es la interoperabilidad con los sistemas de Periférico Universal para Radio de Software (USRP, por sus siglas en inglés). Los USRP son altamente utilizados como radios reconfigurables (Akeela y Dezfouli, 2018). Los USRP en conjunto con GNU Radio han permitido realizar múltiples estudios en el área de radio (Tucker y Tagliarini, 2009).

El uso de SDR se ha convertido en un recurso esencial para los ingenieros que trabajan en el desarrollo y prueba de sistemas de comunicación. Utilizar esta herramienta en el contexto educativo universitario alinea las competencias específicas que debe desarrollar el estudiante con los retos que se encontrará en el mercado laboral. La utilización del SDR como recurso didáctico debe ir en conjunto con una metodología que permita al estudiante estar activo en su proceso de aprendizaje y contribuya a la capacidad de aprendizaje permanente.

\section{Metodología}

Para el desarrollo del estudio se utilizó el método de investigación-acción. El propósito principal de esta metodología es el mejoramiento continuo en la práctica dentro de un contexto natural y no en

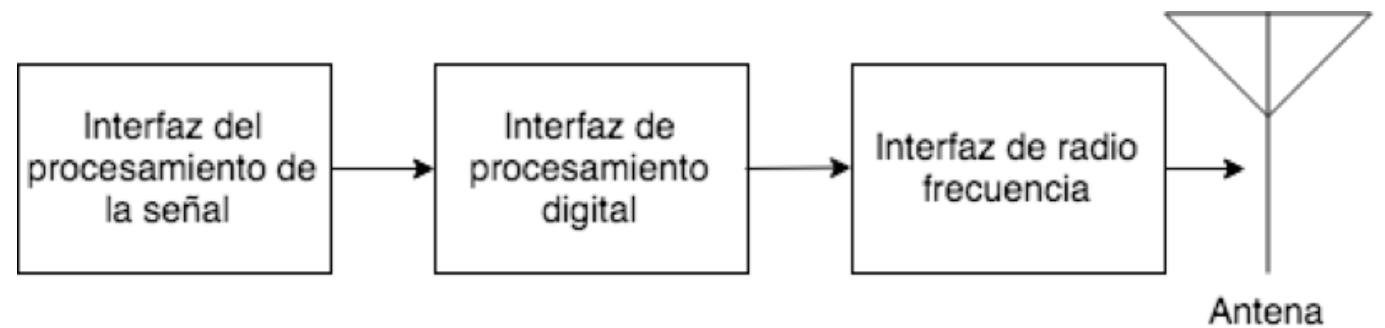

(a) Transmisor

Antena

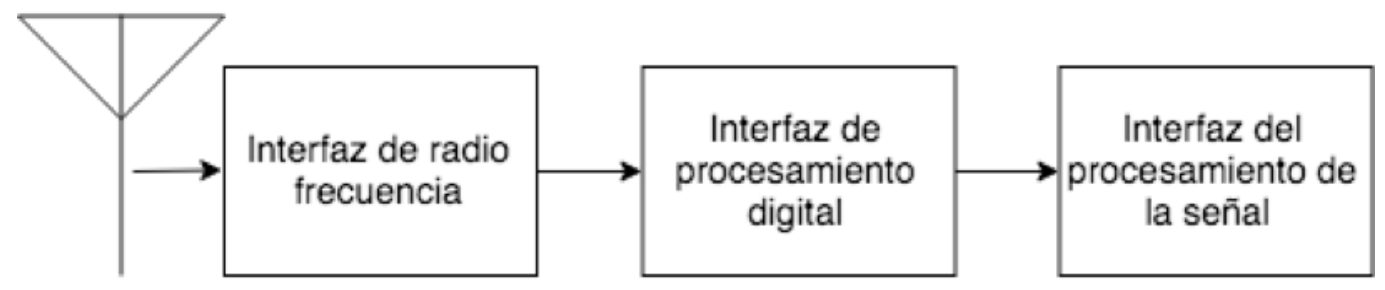

Antena

(b) Receptor 
un ambiente controlado. La investigación-acción se realiza en conjunto con el sujeto de estudio y no en él. La metodología se basa en un ciclo iterativo donde (1) se desarrolla un plan de acción para mejorar el estado actual, (2) se implementan las acciones del plan de mejora, (3) se observan los efectos dentro del contexto donde ocurren y (4) se reflexiona en los efectos para la modificación del plan en las próximas iteraciones del ciclo (Case y Light, 2011). Esta metodología es efectiva en el ámbito educativo ya que permite analizar de forma sistemática las prácticas pedagógicas y, al mismo tiempo, implementar cambios sustanciales (Case y Light, 2011).

Este trabajo se basó en un proyecto de investigación-acción dentro de la asignatura de Electrónica de Comunicación (ITT-233), la cual se imparte en el segundo año del programa académico de Ingeniería Telemática en la Pontificia Universidad Católica Madre y Maestra (PUCMM). La asignatura busca que los estudiantes desarrollen las competencias de diseño de sistemas de comunicación. El diseño de un sistema está impulsado por una necesidad que se refleja en un problema. El diseño y la solución de problemas son competencias cognitivas entrelazadas que requiere poder definir el problema, generar distintas soluciones, determinar el rendimiento o eficiencia de las soluciones, elegir la solución más conveniente con relación a los requerimientos, verificar la solución y evaluar los resultados (Sanz de Acedo, 2010).

Tradicionalmente, la asignatura se ha impartido utilizando metodologías de enseñanza-aprendizajes pasivas y componentes electrónicos como base del diseño y despliegue de los sistemas de comunicación. El uso de estos componentes agrega pasos adicionales que aumentan el tiempo de elaboración de las prácticas que deben lograr los estudiantes al conectar cables entre componentes y solucionar problemas que ocurren en las señales entre dichos componentes. Al mismo tiempo, estos sistemas en el ámbito profesional ya no se diseñan a partir de componentes electrónicos. La industria de la telecomunicación ha pasado a diseñar dentro del contexto de SDR.

La asignatura se planificó para implementar el ABP como una metodología activa de enseñanza-aprendizaje y el SDR como recurso didáctico. En la primera iteración del curso (2017-2018) se utilizó únicamente la plataforma de GNU Radio (v3.7). En la segunda iteración (2018-2019) se utilizó la plataforma de GNU Radio (v3.7) en conjunto con equipos USRP (NI USRP 2920).

Para el apoyo de la metodología, se utilizó un Sistema de Gestión del Aprendizaje (SGA). Estos sistemas son convenientes para orientar al estudiante en los temas a tomar en consideración cuando deben solucionar un problema planteado. Además, ayuda en la comunicación entre estudiantes y el profesor ( Krithivasan et al., 2014). La implementación siguió un esquema parecido al de González, Ferreira y Barranco (2018), donde se utilizó la plataforma Moodle 3.0, la cual es la herramienta que proporciona la universidad. Dentro de la plataforma se colocaron distintos recursos didácticos elaborados por el profesor, al igual que referencias a libros, videos y artículos de investigación. De la misma forma, se utilizó la plataforma para impartir pruebas cortas y dos pruebas de desarrollo. Las soluciones a los problemas planteados debían ser mostradas al profesor y a sus compañeros en fechas establecidas y luego, los archivos correspondientes se colgaban en la plataforma. En la tabla 1 se presenta un resumen de la metodología implementada.

\section{Etapas de los problemas planteados}

En la asignatura se plantearon cinco problemas para ser solucionados en un periodo de 14 semanas. Cada problema tiene un objetivo alineado a la competencia que se desea desarrollar. Estos problemas son abiertos dentro del contexto de la asignatura y se presentaron mal definidos y de forma desestructurada. Se formaron grupos de cuatro estudiantes para poder recurrir a decisiones grupales y poder mejorar la capacidad de toma de decisiones y solución de problemas (Sanz de Acedo, 2010).

Antes de iniciar con los problemas se presentó una práctica con el propósito de familiarizar a los estudiantes con GNU Radio. Para esto se les solicitó que generaran distintos tipos de señales periódicas (triangular, cuadradas, etc.), y que usaran filtros para limitar las armónicas generadas. Se asignó un periodo de dos semanas para la realización de esta práctica.

El primer problema se enfocó en el desarrollo de un sistema de modulación por amplitud (AM) el cual debía manejar distintos tipos de señales, incluyendo las frecuencias de voz que van de $300 \mathrm{~Hz}$ a $3400 \mathrm{~Hz}$. El segundo problema requería que los 
grupos redujeran a la mitad el ancho de banda utilizado por el sistema anterior. Es decir, una mayor eficiencia en el uso del ancho de banda.

El tercer problema solicitaba el diseño de un nuevo sistema utilizando modulación angular (FM o PM). Este sistema debía manejar señales dentro del rango de frecuencias de los sonidos que son audibles para el humano $(20 \mathrm{~Hz}$ a $20 \mathrm{KHz})$. Para el cuarto problema debían enviar dos canales de información simultáneamente y tenían que ser compatibles con el receptor del sistema del problema tres. Es decir, debían duplicar la capacidad de transmisión bajo el mismo requerimiento de ancho de banda del problema anterior.

El último problema se planteó con el esquema de multiplexación ortogonal por división de frecuencia (OFDM, por sus siglas en inglés), el cual forma la base de codificación de señal para los distintos estándares de comunicación utilizados hoy en día, por ejemplo, en WiFi y LTE. Esto presentó una introducción a la base de múltiples estándares, creando un inicio para que se pueda seguir utilizando SDR en asignaturas siguientes que se enfocan en el diseño de sistemas de comunicación más complejos.

Tabla 7. Resumen de la metodología implementada

\begin{tabular}{|c|c|c|}
\hline & $\begin{array}{c}\text { PRIMERA ITERACIÓN } \\
(2017-2018)\end{array}$ & $\begin{array}{c}\text { SEGUNDA ITERACIÓN } \\
(2018-2019)\end{array}$ \\
\hline Fecha & Septiembre 2017 - Diciembre 2017 & Septiembre 2018 - Diciembre 2018 \\
\hline Número de grupos & 4 grupos & 5 grupos \\
\hline Recursos didácticos & GNU Radio v3.7 & $\begin{array}{l}\text { GNU Radio v3.7 } \\
\text { NI USRP } 2920\end{array}$ \\
\hline 1) Desarrollo plan de acción & $\begin{array}{l}\text { Realizado en agosto } 2017 \text {, } \\
\text { Se identificaron las competencias a desa- } \\
\text { rrollar y los recursos didácticos disponibles. } \\
\text { Además, se desarrollaron los problemas } \\
\text { planteados y se calendarizó con relación al } \\
\text { periodo académico. }\end{array}$ & $\begin{array}{l}\text { Realizado en agosto } 2018 \text {, } \\
\text { Se tomaron en consideración las observa- } \\
\text { ciones de incorporar el USRP. Se modificaron } \\
\text { los problemas para que la solución pudiera } \\
\text { incluir el uso de este recurso. }\end{array}$ \\
\hline 2) Implementación del plan & $\begin{array}{l}\text { Realizado en agosto 2017, } \\
\text { Se colocaron distintos recursos didácticos } \\
\text { elaborados y los problemas a resolver dentro } \\
\text { del SGA. En la SGA se programaron las fe- } \\
\text { chas de entrega de cada problema. }\end{array}$ & $\begin{array}{l}\text { Realizado en agosto } 2018 \text {, } \\
\text { Se colocaron distintos recursos didácticos } \\
\text { elaborados y los problemas a resolver dentro } \\
\text { del SGA. En la SGA se programaron las fe- } \\
\text { chas de entrega de cada problema. }\end{array}$ \\
\hline 3) Observación & $\begin{array}{l}\text { Realizado entre septiembre y diciembre } \\
2017 \text {, } \\
\text { Se le dio un seguimiento continuo a cada } \\
\text { grupo y se retroalimentó el trabajo realizado. } \\
\text { Se tomó en consideración las dificultades } \\
\text { que presentaron los grupos y el tiempo que } \\
\text { tomó el trabajo. }\end{array}$ & $\begin{array}{l}\text { Realizado entre septiembre y diciembre } \\
2018, \\
\text { Se le dio un seguimiento continuo a cada } \\
\text { grupo y se retroalimentó el trabajo realizado. } \\
\text { Se tomó en consideración las dificultades } \\
\text { que presentaron los grupos y el tiempo que } \\
\text { tomó el trabajo. }\end{array}$ \\
\hline 4) Reflexión de la implementación & $\begin{array}{l}\text { Realizado en enero 2018, } \\
\text { Se identificó la necesidad de utilizar un re- } \\
\text { curso didáctico que permitiera transmitir las } \\
\text { señales de forma física (USRP). Se consideró } \\
\text { que el manejo del tiempo fue el correcto. }\end{array}$ & $\begin{array}{l}\text { Realizado en enero } 2019 \text {, } \\
\text { Se identificó la necesidad de incorporar com- } \\
\text { petencias blandas en una próxima iteración } \\
\text { y una modificación a la forma de evaluación } \\
\text { correspondiente al cambio. }\end{array}$ \\
\hline
\end{tabular}




\section{Evaluación de los problemas}

Los problemas se evaluaron en dos aspectos: (1) la capacidad de solución de problema y (2) el desempeño de sus diseños.

El primer aspecto se midió verificando de forma presencial que las soluciones cumplieran con los requerimientos expuestos por el problema. Los estudiantes debían hacer una presentación de su solución. Para la medición de desempeño del diseño nos enfocamos en la organización de los bloques en GNU Radio y de la fidelidad de la señal recibida. La fidelidad de una señal se define en la similitud de la señal de salida con relación a la señal de entrada. La fidelidad fue determinada por la calidad del sonido en la presentación de los estudiantes.

\section{Resultados y discusión}

\section{Capacidad de solución de problema}

En la primera iteración (2017-2018), en la cual se usó únicamente GNU Radio, todos los grupos pudieron realizar los cinco (5) problemas planteados de forma exitosa. En la segunda iteración (2018-2019), con GNU Radio y los USRP, todos los grupos pudieron realizar los primeros 4 problemas de forma exitosa con ambos recursos. En el quinto problema, dos grupos pudieron realizar el problema con GNU Radio y los USRP y tres utilizando solamente GNU Radio. La tabla 2 presenta los problemas planteados, el resultado de la capacidad de solución de problemas por iteración y el tiempo asignado para la solución.

La introducción de los USRP en la segunda iteración aumentó el grado de complejidad en los diseños, pero permitió que los estudiantes pudieran observar el comportamiento de sus diseños al transmitir a distintas distancias. El aumento en la complejidad de los diseños con los USRP fue lo que impidió que todos los grupos pudieran completar el quinto problema usando ambas plataformas. Dada esta dificultad y limitación en tiempo, se les permitió que completaran el problema con el uso exclusivo de GNU Radio.

\section{Desempeño del diseño}

En la primera iteración de la asignatura, los grupos presentaron resultados adecuados a los problemas planteados. La capacidad de diseño se reflejó en la organización de los bloques, la figura 2 presenta la organización de los bloques de uno de los grupos, la cual refleja el promedio en organización.

Para la segunda iteración, la distancia entre dos USRP tenía un efecto directo en la fidelidad de la señal por el ruido que entra al canal. La calidad del diseño del sistema permitió que algunos grupos pudieran transmitir a distancias más lejanas. El poder comparar cuál grupo podía transmitir más lejos creó un sentido de competencia que impulsó

Tabla 2. Capacidad de solución de problemas

\begin{tabular}{|l|l|l|c|}
\hline & \multicolumn{1}{|c|}{ PRIMERA ITERACIÓN } & \multicolumn{1}{|c|}{ SEGUNDA ITERACIÓN } & TIEMPO \\
\hline $\begin{array}{l}\text { P1: Sistema AM para señal } \\
\text { de voz }\end{array}$ & $\begin{array}{l}\text { Realizado por } 4 \text { grupos en } \\
\text { GNU Radio }\end{array}$ & $\begin{array}{l}\text { Realizado por } 5 \text { grupos en } \\
\text { GNU Radio + USRP }\end{array}$ & 2 semanas \\
\hline $\begin{array}{l}\text { P2: Sistema AM para señal de } \\
\text { v0z mitad del ancho de banda }\end{array}$ & $\begin{array}{l}\text { Realizado por } 4 \text { grupos en } \\
\text { GNU Radio }\end{array}$ & $\begin{array}{l}\text { Realizado por } 5 \text { grupos en } \\
\text { GNU Radio + USRP }\end{array}$ & 2 semanas \\
\hline $\begin{array}{l}\text { P3: Sistema FM o PM para } \\
\text { señal de audio }\end{array}$ & $\begin{array}{l}\text { Realizado por } 4 \text { grupos en } \\
\text { GNU Radio }\end{array}$ & $\begin{array}{l}\text { Realizado por } 5 \text { grupos en } \\
\text { GNU Radio + USRP }\end{array}$ & 3 semanas \\
\hline $\begin{array}{l}\text { P4: Sistema FM o PM para } \\
\text { doS señales de audio }\end{array}$ & $\begin{array}{l}\text { Realizado por } 4 \text { grupos en } \\
\text { GNU Radio }\end{array}$ & $\begin{array}{l}\text { Realizado por } 5 \text { grupos en } \\
\text { GNU Radio + USRP }\end{array}$ & 3 semanas \\
\hline P5: Sistema OFDM & RNU Radio por 4 grupos en & $\begin{array}{l}\text { Realizado por 2 grupos en } \\
\text { GNU Radio + USRP } \\
\text { Realizado por 3 grupos en } \\
\text { GNU Radio }\end{array}$ & \\
\hline
\end{tabular}


la motivación de los grupos a usar los recursos de forma más eficiente, es decir, un mejor diseño.

\section{Conclusión}

En la Ingeniería Telemática tradicionalmente se han desarrollado las competencias necesarias con el diseño de sistemas de comunicaciones utilizando componentes electrónicos. Con el interés de documentar el desarrollo de un plan de implementación para realizar un cambio al uso de software para el diseño de sistemas de comunicación, se utilizó la metodología ABP y el SDR como recurso didáctico. Esta combinación no solo ayuda a desarrollar las distintas capacidades de diseño, sino que también alinea al estudiante a la forma de trabajar y lo familiariza con las herramientas que utilizará una vez concluya sus estudios universitarios.

Al mismo tiempo, el uso del ABP en conjunto con SDR aumentó el grado de complejidad del diseño de los estudiantes y proporcionalmente, el grado de satisfacción en sus resultados. En las dos iteraciones que ha tenido la asignatura a partir del ABP se puede notar una mayor motivación de los estudiantes cuando se utilizan de forma conjunta la plataforma de GNU Radio con los USRP. Se puede concluir que el uso del ABP en conjunto con SDR presenta una ventaja significativa en comparación a metodologías tradicionales y el uso de componentes electrónicos para el diseño de sistemas de comunicación.

En la asignatura de Electrónica de Comunicación se propusieron problemas abiertos, mal definidos y de forma desestructurada, en los cuales se pudo identificar las tres etapas iterativas de la metodología del ABP. Con cada problema los estudiantes identificaron los conocimientos que necesitaban, realizaron estudios autodirigidos de investigación y aplicaron lo adquirido para su solución. Cada grupo presentó soluciones de diseño distintas por el uso de problemas abiertos, como indican los fundamentos del ABP. Cuando se postula un problema

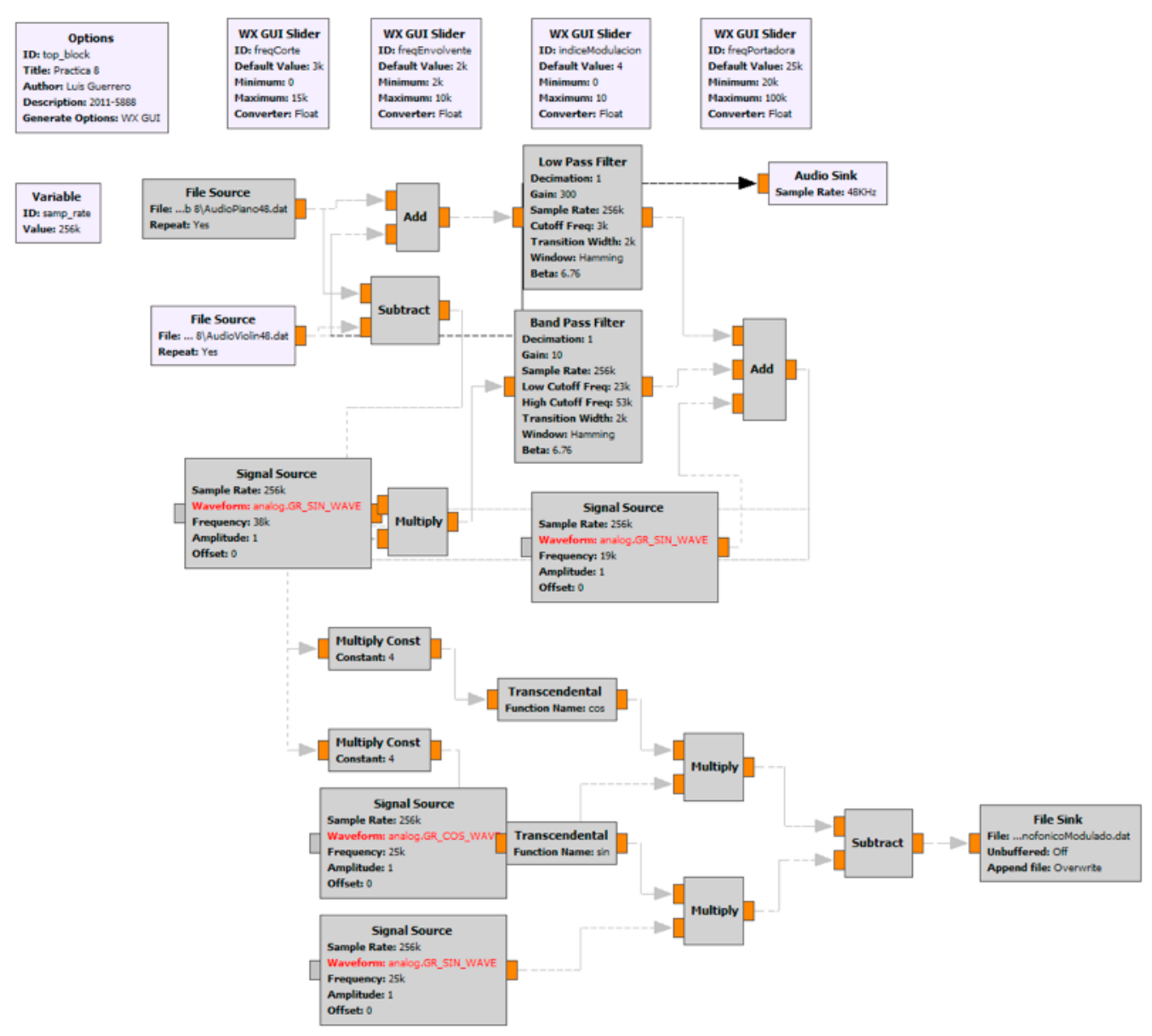

Figura 2. Transmisor de FM dos canales en GNU Radio (Problema 4) 
y se detallan los pasos del mismo, la soluciones planteadas tienden a ser similares por la limitación en las decisiones grupales ya que el problema da indicaciones de la decisión a tomar.

Con la implementación del ABP en esta asignatura el rol del profesor cambió y su principal función consistió en la elaboración de buenos problemas orientados a las competencias que se deseaba desarrollar. Además, pasó a ser un moderador de discusiones con cada problema planteado para ayudar a los estudiantes a definir cada problema, buscar distintas soluciones, seleccionar la mejor solución y evaluar la solución final.

Estos cambios tienen un impacto directo en el manejo del tiempo de los estudiantes y del profesor. En las dos iteraciones de la asignatura pudimos observar diferencias en el manejo del tiempo de los distintos grupos. Es de alta importancia que el profesor tenga la flexibilidad de modificar las fechas de entrega si es necesario. Esto implica que el profesor debe dar un seguimiento continuo a cada grupo para determinar si el esfuerzo que están realizando para solucionar el problema amerita un cambio en la entrega o una modificación del problema, como sucedió con el problema 5 en la segunda iteración de la asignatura.

La metodología del ABP implica que el sistema de evaluación sea transformado conforme al esfuerzo que está llevando a cabo el estudiante. La evaluación debe ser continua y con retroalimentación frecuente para mantener la motivación de los estudiantes. En nuestro caso, todos los problemas tenían la misma ponderación. Sería interesante ver cómo el uso de distintas ponderaciones dependiendo de la complejidad del problema contribuye con la motivación de resolución por parte del estudiante.

La ventaja principal de la investigación-acción es que al ser iterativa permite la mejora continua de los procesos de enseñanza-aprendizaje. Una tercera iteración debe buscar una mayor integración al desarrollo de competencias blandas necesarias para la práctica de ingeniería como el liderazgo, manejo del tiempo y la comunicación. En adición, se pudieran integrar nuevas metodologías como el Aprendizaje-Servicio para acercar los problemas planteados a necesidades sociales. Estos nuevos componentes implicarían cambios a los problemas planteados y al sistema de evaluación.
Para mantener la motivación de los estudiantes hacia su carrera es importante que todas las asignaturas siguientes apliquen metodologías de enseñanza-aprendizaje activas en conjunto con recursos didácticos que utilicen herramientas del ámbito profesional. -

\section{Referencias}

Aguayo Gonzalez, C. R., Dietrich, C. B., Sayed, S., Volos, H. I., Gaeddert, J. D., Robert, P. M., y Kragh, F. E. (2009). Open-source SCA-based core framework and rapid development tools enable software-defined radio education and research. IEEE Communications Magazine, 47(10), 48-55.

Akeela, R., y Dezfouli, B. (2018). Software-defined Radios: Architecture, state-of-the-art, and challenges. Computer Communications, 128, 106-125.

Bejarano, M. T., y Lirio, J. (2008). La utilización de problemas auténticos en la enseñanza superior. El Aprendizaje Basado En Problemas (ABP): Una Propuesta Metodológica En Educación Superior, 35-52. Madrid: Narcea.

Burgess, K. L. (2004). Is your case a problem? Journal of STEM Education: Innovations and Research, 5(1/2), 42.

Case, J. M., y Light, G. (2011). Emerging Research Methodologies in Engineering Education Research. Journal of Engineering Education, 100(1), 186-210.

El-adaway, I., Pierrakos, O., y Truax, D. (2014). Sustainable construction education using problem-based learning and service learning pedagogies. Journal of Professional Issues in Engineering Education and Practice, 141(1), 05014002.

Ertmer, P. A., y Stepich, D. A. (2005). Instructional design expertise: How will we know it when we see it? Educational Technology Research and Development: ETR y $D$, 38-43.

García, A. L., Barros, A. F., Gusso Lenzi, K., Pedroso Meloni, L. G., y Barbin, S. E. (2012). Introduction to the Software-defined Radio Approach. IEEE Latin America Transactions, 10(1), 1156-1161.

Gómez, B. R. (2005). Aprendizaje basado en problemas (ABP): una innovación didáctica para la enseñanza universitaria. Educación $Y$ Educadores. Recuperado de https://dialnet. unirioja.es/servlet/articulo?codigo $=2040741$ 
González, V., Ferreira, J. y Barranco, A. (2018). Desarrollo de habilidades blandas y el uso del Sistema de Gestión del Aprendizaje en la elaboración de proyectos prácticos en una asignatura introductoria de Ingeniería Telemática. Cuaderno de Pedagogía Universitaria, Vol. 15, n. $^{\circ} 29$, pp. $44-53$

Grolinger, K. (2011). Problem Based Learning in Engineering Education: Meeting the needs of industry. Teaching Innovation Projects, 1(2). Recuperado de https://ojs.lib.uwo.ca/index. php/tips/article/view/3564

Harun, N. F., Yusof, K. M., Jamaludin, M. Z., y Hassan, S. A. H. S. (2012). Motivation in Problem-based Learning Implementation. Procedia - Social and Behavioral Sciences, 56, 233-242.

Hmelo-Silver, C. E. (2013). Creating a Learning Space in Problem-based Learning. Interdisciplinary Journal of Problem-Based Learning, 7(1), 5.

Jondral, F. K. (2005). Software-defined Radio: Basics and Evolution to Cognitive Radio. EURASIP Journal on Wireless Communications and Networking, 2005(3), 275-283.

Krithivasan, S., Shandilya, S., Arya, K., Lala, K., Manavar, P., Patii, S., y Jain, S. (2014). Learning by competing and competing by learning: Experience from the e-Yantra Robotics Competition. 2014 IEEE Frontiers in Education Conference (FIE) Proceedings, 1-8.

Küçük, K. (2018). RTWiFi-Lab: A real-time Wi-Fi laboratory platform on USRP and LabVIEW for wireless communications education and research. Computer Applications in Engineering Education, 26(1), 111-124.

Leary, H., Walker, A., y Shelton, B. E. (2013). Exploring the relationships between tutor background, tutor training, and student learning: A problem-based learning meta-analysis. Interdisciplinary Journal of Problem-Based Learning, 7 (1). Recuperado de https://docs.lib.purdue.edu/ijpbl/vol7/iss1/6/

Masek, A., y Yamin, S. (2012). A Comparative Study of the Effect of Problem Based Learning and Traditional Learning Approaches on Students' Knowledge Acquisition. International Journal of Engineering Education, 28(5), 1161.

Núñez, J. M., y Mascareñas y Pérez-Iñigo, C. (2016). Software defined radio (SDR) on radiocommunications teaching. Recuperado de http://rodin.uca.es/xmlui/handle/10498/18718

Patil, R. T. (2016). Comparative Study of Problem Based Learning and Traditional Lecture Delivery-A Case Study. Journal of Engineering Education Transformations. Recuperado de https://doi.org/10.16920/jeet/2016/v0i0/111641
Patton, L. K. (2007). A GNU Radio Based SoftwareDefined Radar (Wright State University). Browse all Theses and Dissertations. 91. Recuperado de https://corescholar.libraries.wright.edu/etd_all/91/

Perrenet, J. C., Bouhuijs, P. A. J., y Smits, J. G. M. M. (2000). The Suitability of Problem-based Learning for Engineering Education: Theory and practice. Teaching in Higher Education, 5(3), 345-358.

Rico, R., y Ertmer, P. A. (2015). Examining the Role of the Instructor in Problem-centered Instruction. TechTrends, 59(4), 96-103.

Sanz de Acedo, M. L. (2010). Competencias cognitivas en Educación Superior. Madrid: Narcea Ediciones.

Savery, J. R. (2006). Overview of problem-based learning: definition and distinctions, The interdisciplinary. Journal of Problem-Based Learning. Recuperado de http://citeseerx.ist.psu. edu/viewdoc/summary?doi=10.1.1.557.6406

Tan, C. P., Van der Molen, H. T., y Schmidt, H. G. (2016). To what extent does problem-based learning contribute to students' professional identity development? Teaching and Teacher Education, 54, 54-64.

Tucker, D. C., y Tagliarini, G. A. (2009). Prototyping with GNU radio and the USRP - where to begin. IEEE Southeastcon 2009, 50-54.

Yadav, A., Subedi, D., Lundeberg, M. A., y Bunting, C. F. (2011). Problem-based learning: Influence on students' learning in an electrical engineering course. Journal of Engineering Education, 100(2), 253-280. 